\title{
Voice Controlled Wheelchair Based on a Tmega
}

\author{
Kamlesh Kukreti, Raghav Chabbra, Aditya ,Rahul Rawat, Vijay Kumar
}

\begin{abstract}
The dependency of people depends on others especially when they get to travel from one place to another. For those who are physically ill requires wheelchair for their daily needs, and who can help them to make the wheelchair move. By having a controlled wheel-chair system will empower weakened individuals to wind up independent. The structure is a remote wheelchair control system that uses a voice acknowledgment application for actuating and controlling all of its improvements. The wheelchair responds to the voice request from its customers to play out any advancement limits. It joins a microcontroller, Bluetooth module, control interface engine, transfer engine, which uses board through which wheel chair can move. By using the structure, the customers can work in the wheel-chair by basically address the mobile phone antenna. The fundamental improvement limits fuse advance and alter heading, left and right turns and stop It utilizes an Atmega328 to control the system assignments. It talks with the Bluetooth module to recognize word communicated and after that chooses the contrasting yield request with drive, the right and left engines. To accomplish this endeavour, in the controller's memory a low-level registering program is set.
\end{abstract}

Index Terms: Arduino, Bluetooth module HC-05, L293D, Voice application, MASTER/SLAVE.

\section{INTRODUCTION}

The people with disabilities can be happy with powered wheelchair, but people with the crippled network, found out that working a power wheelchair is somewhat difficult or troublesome. This undertaking could be a piece of an assistive innovation. It is for progressively autonomous, beneficial, and agreeable living. An incapacitated individual who is having movement related issues or locomotive disabilities require a wheelchair so that he/she can move around and perform different tasks. This can be done by moving the wheelchair by hands. At any rate various individuals have feeble upper limbs or find the manual technique for working unreasonably tiring. Thus it is more appealing to provide them such sort of a wheelchair that can be moved or controlled by giving voice direction or using a joystick. Since this wheelchair can move at a good reasonable speed, it has that capacity that it can maintain distance from hindrances. This all can be accomplished at a very moderate cost, which will be a great boon to all those incapacitated people out there. In addition, it is also good for those organizations that come up to help these people.

Revised Manuscript Received on April 25, 2019. India. E-mail: kamlesh.kukreti23@gmail.com

Raghav Chabbra, Graphic Era Deemed to be University, Dehradun, India.

Aditya, Graphic Era Deemed to be University, Dehradun, India.

Rahul Rawat, Graphic Era Deemed to be University, Dehradun, India.

Vijay Kumar, Professor, Department of Physics, Graphic Era Hill University, Dehradun, India
Kamlesh Kukreti1, Graphic Era Deemed to be University, Dehradun,

After discussing all these problems and keeping them in mind we proposed an automated wheelchair which has an obstruction shirking capability. All the powered wheelchairs till now use interfaces to work on. This is still insufficient in terms of providing free portability to those who are handicapped. Through structural study and some research work, it was discussed that the powered wheelchair needs some sort of improvement to help those debilitated people out there. It is to be made more self versatile. This exploration paper is going to provide creative answers for handling the wheel seats using voice interface.

The paper is divided into four sections, including the current introductory Section 1. This is followed by Section 2 where the basic methodology for making the automation of the wheelchair has been explained with voice application. Thereafter in section 3 the components setup is discussed for obtaining the results. These components are employed for enhancing the work function of the automation of wheel chair using voice recognition system. After this, concluding remarks have been given in section 4

\section{METHODOLOGY}

Front The examination comprises of Bluetooth module and engine driver with a microcontroller. The entire framework is connected to the seat. In the opposite end one cell phone is joined to the seat with AMR_Voice application. So the versatile will send the voice signs to the Bluetooth module and will be perceived by the microcontroller. There are mostly five capacity modified in a microcontroller forward, in reverse, left, right and stop.

Front two engines are given high flag to move a forward way. Raise two engines are given high flag to move a regressive way. Left two engines are given high flag to move the correct way. Right two engines are given high flag to move in the left course. Low flag is given to each engine to stop.

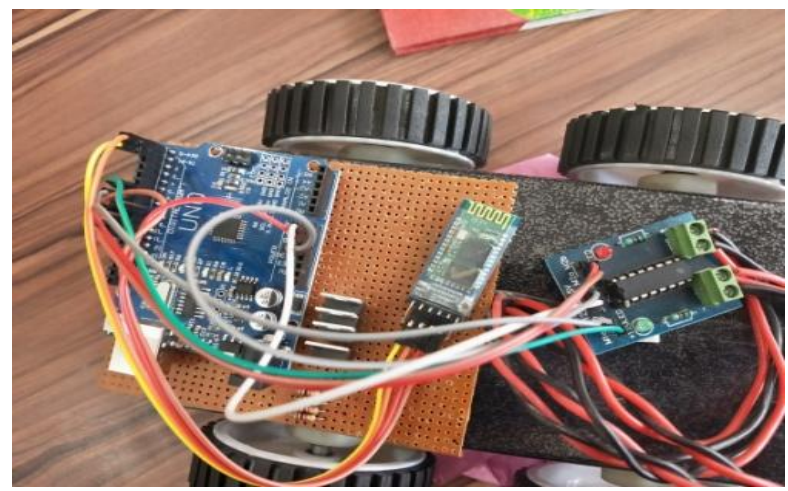

Fig.1. Represent the protype for the project

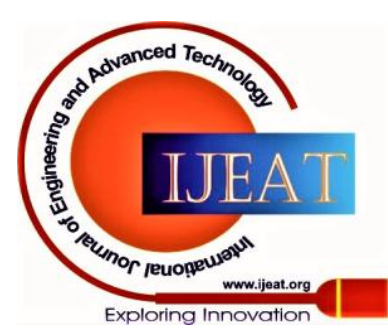


In 'Voice acknowledgment wheelchair' order will be given to the versatile utilizing an application which is interfaced with the Bluetooth module and microcontroller, which will be mounted on the wheelchair The program is put away/consumed in the memory of the microcontroller. There are for the most part five directions perceived by the microcontroller forward, in reverse, right, left. The engine driver is interfaced with the microcontroller. $12 \mathrm{~V}$ power supply is given to the engines. The fundamental piece of the plan is to controlling the movement of the wheel-chair. There are four state of movements that are considered such as, pushing ahead, moving backward course, moving to one side and moving to one side. On the off chance that the client does not need the wheel-chair move in rapid, the moderate speed in order to set by applying current supply to the motor. The wheel seat bearings as development of conceivable are as given beneath and alsoFig.1represent Block Diagram for Voice Controlled Wheel-chair Based on Atmega

Forward Direction: Both the motor are moving in forward direction. Turn around: Both motor are moving in backward way. Left: Left side motor halted and right engine in forward way.Right: Right motor is stopped and left motor moves in forward way direction.Stop: All motors are halted.

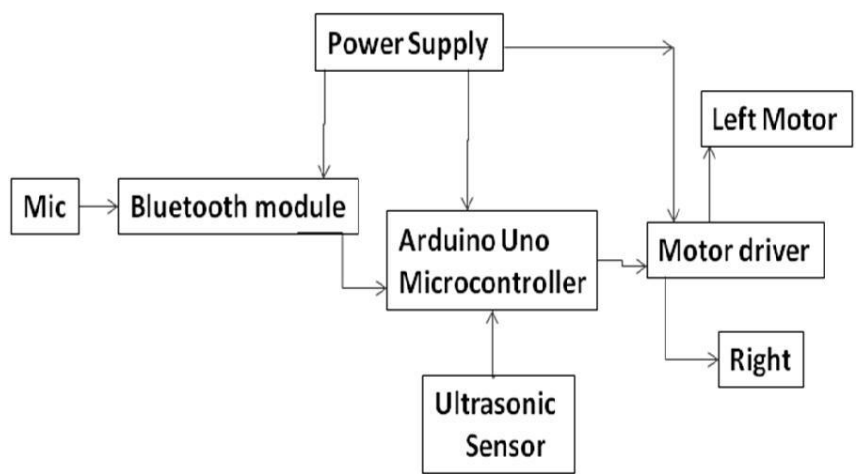

Fig.2 Block Diagram for Voice Controlled Wheelchair Based on AT mega

\section{RELATED WORK}

The works focuses to set up the automated wheel-chair control frame-work in order to recognize voice module at lower cost as in unexpected of crippled individuals particularly with a handicap who cannot make motion like ordinary persons will having the option to move freely. In this venture, a basic bundle with the modest components that wouldn't complicate the wheel-chair and with the high productivitydrawbacks voice recognizer that could accomplish some of the time to $100 \%$ of acknowledgment rate and is utilizes to manufacture, a viable voice-controlled wheel-chair. Fig.2. represent Wheelchair in which circuit has to be implemented and has wider applications as well as Fig.3. Represents Circuit diagram for the project

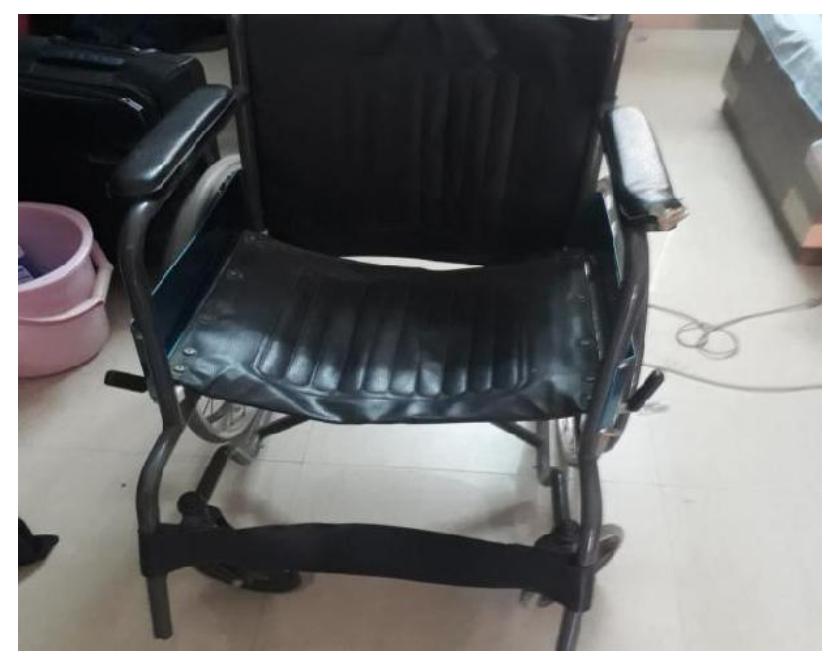

Fig.3. Wheelchair in which circuit must be implemented

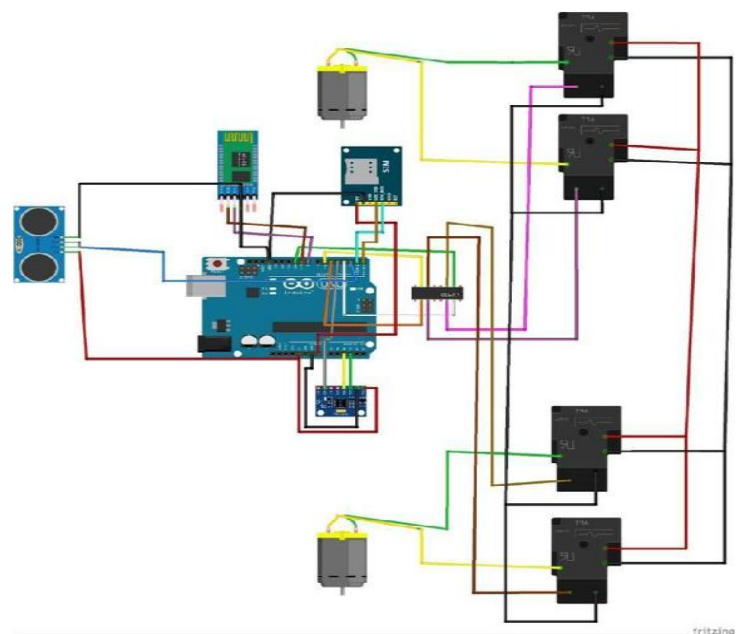

Fig.4. shows the circuit connection for the Voice Controlled Wheelchair Based on Arduino Uno

\section{CONCLUSION}

This paper examinesthis voice worked wheel objective of this research project is to equip the present motorized wheelchair control system with a voice command system at low-price and friendly operation. By having these features, differently abled people especially with a severe disability who are unable to move like normal people will be able to move independently. An individual with crippled with legs and arms can utilize this wheel seat effectively on the off chance that he can talk.

\section{REFERENCES}

1. R.Z. Raiyan, M. S. Nawaz, A. K. M. A. Adnan and M. H. Imam, "Design of an arduino based voice-controlled automated wheelchair," 2017 IEEE Region 10 Humanitarian Technology Conference (R10-HTC), Dhaka, 2017, pp. 267-270.

2. Azam, G., and M. T. Islam. "Design and Fabrication of a Voice Controlled Wheelchair for Physically Disabled People"

3. Anusha, S., M. Madhavi, and R. Hemalatha. "HOME AUTOMATION USING ATmega328 MICROCONTROLLER AND ANDROID APPLICATION." (2015).

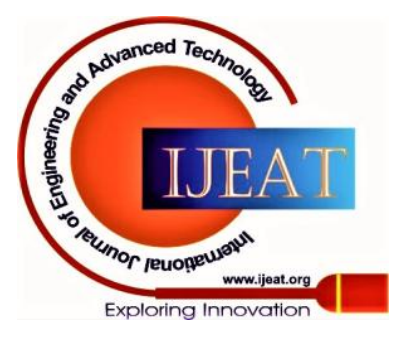


4. Megalingam, Rajesh Kannan et al. "'Gest-BOT'-A Highly Convenient Locomotive Solution for the Elderly and Physically Challenged." Global Humanitarian Technology Conference (GHTC), 2012 IEEE. IEEE, 2012.

5. Skraba, Andrej, et al. "Prototype of speech controlled cloud based wheelchair platform for disabled persons." Embedded Computing (MECO), 2014 3rd Mediterranean Conference on. IEEE, 2014.

6. Sobia, M. Carmel, V. Brindha, and A. Abudhahir. "Facial expression recognition using PCA based interface for wheelchair." Electronics and Communication Systems (ICECS), 2014 International Conference on. IEEE, 2014.

7. Klabi I., Masmoudi M.S., Masmoudi M., "Advanced user interfaces for intelligent wheelchair system", 1st IEEE Conference on Advanced Technologies for Signal and Image Processing, 2014 pp.130-136, Tunisia

8. Rajini, Gangadhari and Lr Siva. "Android MobilePhone Controlled Bluetooth Robot Using Arm7 Microcontroller." (2015).

9. Kevin Brady, Michael Brandstein, Thomas Quatieri, Bob Dunn "An Evaluation Of Audio-Visual person Recognition on the XM2VTS corpus using the Lausanne protocol" MIT Lincoln Laboratory, 244 Wood St., Lexington MA

10. W. M. Campbell_, D. E. Sturim W. Shen D. A. Reynolds_, J. Navr'atily "The MIT- LL/IBM Speaker recognition System using High performance reduced Complexity recognition" MIT Lincoln Laboratory IBM 2006.

11. ZahiN.Karam,WilliamM.Campbell "A new Kernel for SVM MIIR based Speaker recognition "MIT Lincoln Laboratory, Lexington, MA, USA.

12. Asghar. Taheri ,Mohammad Reza Trihi et.al,Fuzzy Hidden Markov Models for speech recognition on based FEM Algorithm, Transaction on engineering Computing and Technology V4 February 2005,IISN,1305-5313

13. GIN-DER WU AND YING LEI “A Register Array based Low power FFT Processor for speech recognition" Department of Electrical engineering national Chi Nan university Puli ,545 Taiwan. 